\title{
Resolution Pyramids on the FCC and BCC Grids
}

\author{
Robin Strand ${ }^{1}$ and Gunilla Borgefors ${ }^{2}$ \\ 1 Centre for Image Analysis, Uppsala University, \\ Lägerhyddsvägen 3, SE-75237 Uppsala, Sweden \\ 2 Centre for Image Analysis, Swedish University of Agricultural Sciences, \\ Lägerhyddsvägen 3, SE-75237 Uppsala, Sweden \\ \{robin, gunilla\}@cb.uu.se
}

\begin{abstract}
Partitionings on the face-centered cubic grid and the bodycentered cubic grid that are suitable for resolution pyramids are found. The partitionings have properties similar to a partitioning that has been used for the resolution pyramids on the cubic grid. Therefore, they are well-suited for adapting methods to construct multiscale representations developed for the cubic grid. Multiscale representations of images are constructed using different methods.
\end{abstract}

\section{Introduction}

Three-dimensional images are usually captured into the cubic grid. Often, the images are computed from projections rather than captured directly. It is, however, possible to adjust image capturing techniques such as CT or MRI to produce images on other grids, such as the face-centered cubic (fcc) and the body-centered cubic (bcc) grids [1]. The sampling theorem allows a decrease of the number of grid points by a factor 1.41 on the bcc grid compared to the cubic grid without influencing the image representation/reconstruction quality, [1]. It has been demonstrated that the hexagonal grid in two dimensions is theoretically better than the square grid, [2]. For example, the hexagonal grid has a higher packing density than the square grid. In three dimensions, the fcc and bcc grids both have higher packing densities than the cubic grid. The fcc and bcc grids are the three-dimensional "equivalents" of the hexagonal grid, [3]. Since it is very easy to construct efficient data structures for the fcc and bcc grids, the only missing piece for allowing full use of the fcc and bcc grids in image processing is to construct efficient algorithms for analysis and processing.

Data structures based on resolution pyramids can be used to improve both the computation time and the quality in image analysis applications, e.g. matching [4] and segmentation [5]. When using the two dimensional square grid or the three dimensional cubic grid, partitioning is straightforward, [6]. The literature on resolution pyramids on the hexagonal grid is rich, e.g., $[7,8,9,10]$. In this article, resolution pyramids on the fcc and bcc grids are examined. 
In the first part of the article, we calculate partitionings that are suitable for resolution pyramids on the fcc and bcc grids. We use conditions similar to those derived for the hexagonal grid in [7]. The most important property is that all levels in the pyramid should be represented by the same grid. The partitionings have many properties in common with the partitioning used on the cubic grid and are therefore well-suited for adapting methods to construct multiscale representations developed for the cubic grid. Two different methods are presented and illustrated using a running example.

\section{The Grids}

In [3], a grid $\mathbb{G}$ is defined as any set of points in $\mathbb{R}^{n}$. In this article, we will use three-dimensional grids on the form:

$$
\mathbb{G}_{T}=\left\{T(x, y, z): x, y, z=a \overrightarrow{v_{1}}+b \overrightarrow{v_{2}}+c \overrightarrow{v_{3}}, a, b, c \in \mathbb{Z}\right\}
$$

where $T$ is an affine transformation. We refer to $\overrightarrow{v_{1}}, \overrightarrow{v_{2}}$, and $\overrightarrow{v_{3}}$ as base vectors. By using this definition, we do not require the origin to be in the grid. We will consider three types of grids:

\section{Definition 1.}

- With vectors $\overrightarrow{v_{1}}=(1,0,0), \overrightarrow{v_{2}}=(0,1,0)$, and $\overrightarrow{v_{3}}=(0,0,1)$, and a rigid transformation $T$ such that (1) is fulfilled, $\mathbb{G}_{T}$ is a cubic grid, denoted $\mathbb{Z}_{T}^{3}$.

- With vectors $\overrightarrow{v_{1}}=(1,0,1), \overrightarrow{v_{2}}=(0,1,1)$, and $\overrightarrow{v_{3}}=(1,1,0)$, and a rigid transformation $T$ such that (1) is fulfilled, $\mathbb{G}_{T}$ is an fcc grid, denoted $\mathbb{F}_{T}$.

- With vectors $\overrightarrow{v_{1}}=(1,-1,1), \overrightarrow{v_{2}}=(-1,1,1)$, and $\overrightarrow{v_{3}}=(1,1,-1)$, and a rigid transformation $T$ such that (1) is fulfilled, $\mathbb{G}_{T}$ is a bcc grid, denoted $\mathbb{B}_{T}$.

If $T=I$ is the identity mapping, then we use the notation $\mathbb{G}=\mathbb{G}_{T}$.

For each grid point in an fcc grid, there are 12 face-neighbours and 6 vertexneighbours, resulting in the 12- and 18-neighbourhoods, see Fig. 1(b),(c). In a bcc grid, the neighbours connected to a grid point are all face-neighbours, see Fig. 1(e),(f). However, there are two kinds of face-neighbours, resulting in the 8-

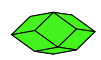

(a)

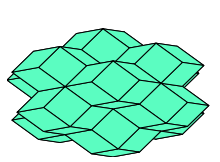

(b)

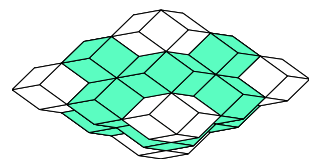

(c)

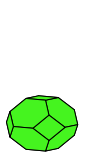

(d)

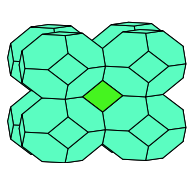

(e)

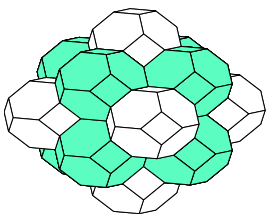

(f)

Fig. 1. A voxel (a) in fcc and its 12- and 18-neighbours, shown in (b) and (c) respectively. A voxel in bcc (d) and its 8- and 14-neighbours, shown in (e) and (f) respectively 
and 14-neighbourhoods. The light-grey voxels in Fig. 1 are referred to as firstorder neighbours and the white voxels as second-order neighbours. Throughout this article, the set of object grid points are visualized by their voxels (the Voronoi regions).

\section{Tessellating the FCC and BCC Grids}

\subsection{Conditions for the Tessellations}

In this section, a way to construct partitionings of the fcc and bcc grids is presented. Note that we are not concerned with tiles in continuous space, but with "patterns" of grid points. The goal is basically to cluster neighbouring grid points in such a way that the centroid of the cluster will form a grid point in the next level in a resolution pyramid, and these new grid points will form an appropiate grid.

There is no standard notation for these clusters. The term "aggregate" is used in [8] to denote a cluster in a resolution pyramid. The term "pattern" is used in [7]. In [9], the same term is used for a matching operation using resolution pyramids. A pattern is defined to be a vector of elements of the set $\{0,1, D\}$, where $D$ indicates a "don't care" position. A centroid of a cluster is often referred to as parent and the elements in the cluster as children. We will adopt the notation used in [7] with a slight modification.

The tessellation of a grid is carried out by clustering grid points together in a pattern. Patterns in the hexagonal grid were examined in [7]. A generator $\mathbf{Q} \subset \mathbb{G}$ is a set of grid points, e.g., as in Fig. 2. By considering translations and rotations of the generator, a larger subset of the grid is covered. The set $\mathcal{P}=\left\{\mathbf{P}_{i}, i \in \mathcal{I}\right\}$, where $\mathcal{I}$ is a set of indices and $\mathbf{P}_{i}$ are the patterns, i.e., translations and rotations of $\mathbf{Q}$ can be constructed such that there is an index $i$ satisfying $\mathbf{P}_{i}=\mathbf{Q}$. The centroids of the patterns will form a new grid at the next level in the resolution pyramid.

Definition 2. The set $\mathcal{P}=\left\{\mathbf{P}_{i}, i \in \mathcal{I}\right\}$ tessellates the grid $\mathbb{G}$ if the following is fulfilled:

$-\mathbb{G}=\bigcup_{i \in \mathcal{I}} \mathbf{P}_{i}$.

- For any two patterns $\mathbf{P}_{i}$ and $\mathbf{P}_{j}, \mathbf{P}_{i} \cap \mathbf{P}_{j}=\varnothing \Longleftrightarrow i \neq j$.

Example 1. Let $\mathbf{Q}=\{(0,0,0),(1,1,1)\}$ (see Fig. 2(b)) be a generator. A set of patterns $\mathcal{P}$ that tessellates $\mathbb{B}$ can be constructed by translating $\mathbf{Q}$ by integer multiples of $(2,0,0),(0,2,0)$, and $(0,0,2)$. If $T$ is a translation from $(0,0,0)$ to the centroid of $\mathbf{Q}$ and a scaling by a factor 2 , then the centroids of the patterns satisfy the conditions for being a cubic grid in Definition 1 . The new grid is $\mathbb{Z}_{T}^{3}$.

\subsection{Finding Admissible Patterns}

If we can construct a set of patterns such that the grid formed by the centroids of the patterns $\mathbb{G}_{T}$ is of the same type as the original grid $\mathbb{G}$, then by repeating the 


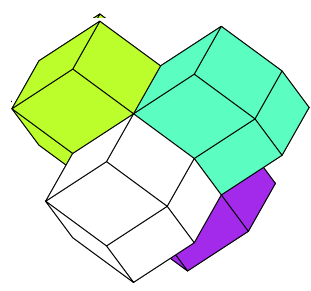

(a)

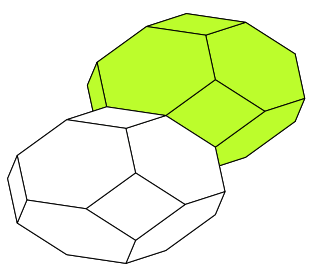

(b)

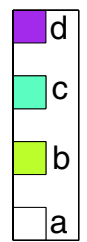

(c)

Fig. 2. Examples of patterns in (a) fcc and (b) bcc. The grid points are color-coded as in (c)

procedure recursively, a resolution pyramid with levels $\mathbb{G}, \mathbb{G}_{T}, \mathbb{G}_{T^{2}}, \ldots, \mathbb{G}_{T^{m}}$ is achieved. We will now calculate patterns that can be applied in such a recursive manner.

Definition 3. Given a grid $\mathbb{G}$, a set of patterns $\mathcal{P}$ is admissible if it fulfills the following conditions:

C1 $\mathcal{P}$ tessellates $\mathbb{G}$.

C2 The grid formed by the centroids of the patterns is of the same type as $\mathbb{G}$.

C3 For any pattern $\mathbf{P}_{i}$, there is a one-to-one translation mapping $S$ between the generator $\mathbf{Q}$ and the pattern $\mathbf{P}_{i}, S: \mathbf{Q} \rightarrow \mathbf{P}_{i}$.

C4 The pattern should be compact in the sense that it should be a connected set.

Conditions C1 and C2 are necessary for a pattern to be used in a resolution pyramid where all levels form the same grid. The one-to-one mapping in C3 will be used when labelling the grid points in the patterns. Moreover, C3 will be used when searching for admissible patterns. Without condition $\mathbf{C 4}$, we might end up in a set of patterns that is not suitable for resolution pyramids; we want a grid point in one level to correspond to a set of connected grid points in the previous level. The patterns obtained by the generators in Fig. 2 satisfy all conditions but C2. In fact, both patterns form a cubic grid.

We denote the first level, the grid we start with, in the pyramid by $\mathbb{G}$. The second level, the grid formed by the centroids of patterns of the original grid, is denoted $\mathbb{G}_{T}$. By repeating this procedure, the resolution pyramid consisting of the levels $\mathbb{G}, \mathbb{G}_{T}, \mathbb{G}_{T^{2}}, \ldots, \mathbb{G}_{T^{m}}$ is achieved.

Suppose that each $\mathbf{P}_{i}$ consists of $n$ grid points. We denote by $\delta_{\mathbb{G}}$ the distance between two first-order neighbours in $\mathbb{G}$. For the fcc and bcc grids $\mathbb{F}$ and $\mathbb{B}$, $\delta_{\mathbb{F}}=\sqrt{2}$ and $\delta_{\mathbb{B}}=\sqrt{3}$. If the set of patterns satisfies $\mathbf{C 1}$, then the new grid, $\mathbb{G}_{T}$, will have $1 / n$ as many grid points as the original, distributed over the same volume. If it also satisfies C2, the distance between first-order neighbours in $\mathbb{G}_{T}$ is $\delta_{\mathbb{G}_{T}}=\sqrt[3]{n} \delta_{\mathbb{G}}$. By $\mathbf{C 1}$, the patterns should also tessellate the grid, therefore $n$ must be an integer. We get the following condition: 
Table 1. The grid points in $\mathbb{G}$ are sorted according to their distance to $\mathbf{0}$. This distance will be the distance between first-order neighbours in $\mathbb{G}_{T}, \delta_{\mathbb{G}_{T}}$. For each distance, condition (2) is tested

\begin{tabular}{|c|c|c|c|c|c|c|c|c|}
\hline \multicolumn{3}{|c|}{ fcc } & \multicolumn{3}{|c|}{ bcc } & \multicolumn{3}{|c|}{ cubic } \\
\hline$\delta_{\mathbb{G}_{T}}$ & $n$ & \# points & $\delta_{\mathbb{G}_{T}}$ & $n$ & \# points & $\delta_{\mathbb{G}_{T}}$ & $n$ & \# points \\
\hline$\sqrt{2}$ & 1 & 12 & $\sqrt{3}$ & 1 & 8 & 1 & 1 & 6 \\
\hline 2 & $2^{\frac{3}{2}}$ & 6 & 2 & $\left(\frac{2}{3}\right)^{\frac{3}{2}}$ & 6 & $\sqrt{2}$ & $2^{\frac{3}{2}}$ & 12 \\
\hline$\sqrt{6}$ & $3^{\frac{3}{2}}$ & 24 & $\sqrt{8}$ & $\left(\frac{8}{3}\right)^{\frac{3}{2}}$ & 12 & $\sqrt{3}$ & $3^{\frac{3}{2}}$ & 8 \\
\hline$\sqrt{8}$ & 8 & 12 & $\sqrt{11}$ & $\left(\frac{11}{3}\right)^{\frac{3}{2}}$ & 24 & 2 & 8 & 6 \\
\hline$\sqrt{10}$ & $5^{\frac{3}{2}}$ & 24 & $\sqrt{12}$ & 8 & 8 & $\sqrt{5}$ & $5^{\frac{3}{2}}$ & 24 \\
\hline$\sqrt{12}$ & $6^{\frac{3}{2}}$ & 8 & 4 & $\left(\frac{16}{3}\right)^{\frac{3}{2}}$ & 6 & $\sqrt{6}$ & $6^{\frac{3}{2}}$ & 24 \\
\hline$\sqrt{14}$ & $7^{\frac{3}{2}}$ & 48 & $\sqrt{19}$ & $\left(\frac{19}{3}\right)^{\frac{3}{2}}$ & 24 & $\sqrt{8}$ & $8^{\frac{3}{2}}$ & 12 \\
\hline 4 & $8^{\frac{3}{2}}$ & 6 & $\sqrt{20}$ & $\left(\frac{20}{3}\right)^{\frac{3}{2}}$ & 24 & 3 & 27 & 30 \\
\hline$\sqrt{18}$ & 27 & 36 & $\sqrt{24}$ & $8^{\frac{3}{2}}$ & 24 & $\sqrt{10}$ & $10^{\frac{3}{2}}$ & 24 \\
\hline$\sqrt{20}$ & $10^{\frac{3}{2}}$ & 24 & $\sqrt{27}$ & 27 & 32 & $\sqrt{11}$ & $11^{\frac{3}{2}}$ & 24 \\
\hline$\sqrt{22}$ & $11^{\frac{3}{2}}$ & 24 & $\sqrt{32}$ & $\left(\frac{32}{3}\right)^{\frac{3}{2}}$ & 12 & $\sqrt{12}$ & $12^{\frac{3}{2}}$ & 8 \\
\hline
\end{tabular}

$$
\left(\frac{\delta_{\mathbb{G}_{T}}}{\delta_{\mathbb{G}}}\right)^{3}=n \in \mathbb{N}
$$

In order to find the admissible patterns, we need to label the grid points in the patterns. We use the labels $\mathbf{a}, \mathbf{b}, \mathbf{c}, \ldots$ as in Fig. 2. Assume that the generator $\mathbf{Q}$ consists of $n$ grid points. Each element in $\mathbf{Q}$ is given a unique label. By C3, for any $i \in \mathcal{I}$, there is a one-to-one mapping involving only translation between $\mathbf{Q}$ and $\mathbf{P}_{i}$. Therefore, each grid point is identified by exactly one of the labels.

Using the one-to-one mapping, it is easy to see that the distance between the centroids of two patterns is equal to the distance between the a-points in the patterns. Let $\mathbf{0}$ be the a-point of the generator $\mathbf{Q}$. To find possible values of $n$, we sort the grid points by their distances to $\mathbf{0}$ and check if (2) is fulfilled. This is done by using $\delta_{\mathbb{G}_{T}}=d(\mathbf{0}, q)$, where $q$ is the a-point in a neighbouring pattern. There might of course be many grid points at the same distance from $\mathbf{0}$. We want $q$ to correspond to a first-order neighbour in the new grid $\mathbb{G}_{T}$. Therefore, there must be at least as many grid points at distance $\delta_{\mathbb{G}_{T}}$ as the number of first-order neighbours in $\mathbb{G}$. For comparison, we include the cubic grid in Table 1.

In Table 1, we see that the smallest admissible patterns on $\mathbb{F}$ have distance $\sqrt{8}$ to the first-order neighbours. The generators are constructed by clustering 8 neighbouring grid points and checking that they satisfy $\mathbf{C 1}-\mathbf{C 4}$. A generator satisfying this is, e.g., $\mathbf{Q}=\{(0,0,0),(1,1,0),(1,-1,0),(1,0,-1),(1,0,1)$, $(0,1,1),(0,1,-1),(2,0,0)\}$. On $\mathbb{B}$, the distance between first-order neighbours of the centroids is $\delta_{\mathbb{G}_{T}}=\sqrt{12}$. We get, e.g., the generator $\mathbf{Q}=\{(0,0,0),(1,1,1)$, $(1,-1,1),(1,1,-1),(1,-1,-1),(2,0,0),(0,2,0),(0,0,2)\}$. Both these set of 


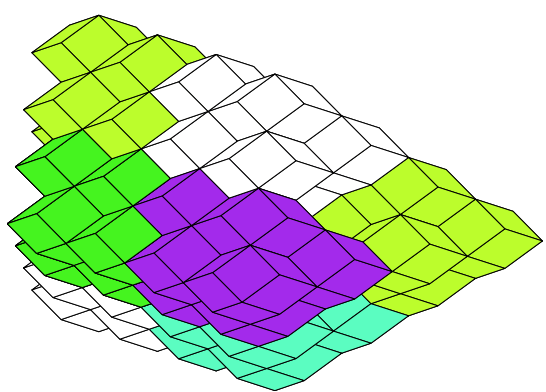

(a)

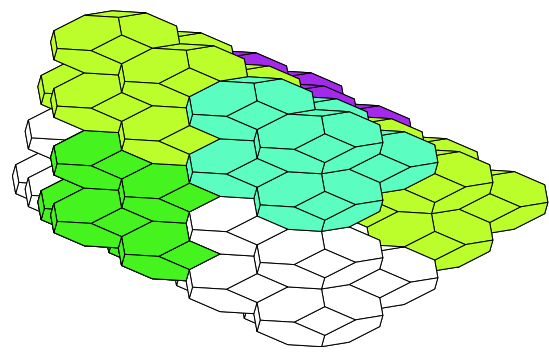

(c)

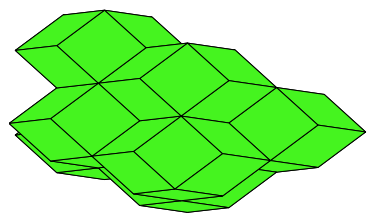

(b)

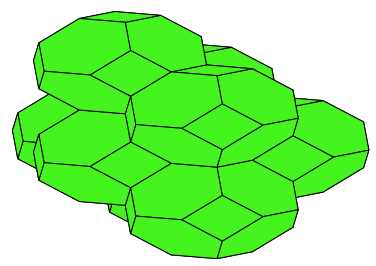

(d)

Fig. 3. A set of grid point in $\mathbb{G}=\mathbb{F},(\mathrm{a})$, and the corresponding set of grid points in $\mathbb{G}_{T},(\mathrm{~b})$. Analogously on a bcc grid, (c) and (d)

patterns satisfy $\mathbf{C 1}-\mathbf{C} 4$ and has the ratio $(1: 8)$, i.e., each pattern consists of 8 grid points. They are shown in Fig. 3. For both sets of patterns, if $T$ is a translation from $(0,0,0)$ to the centroid of $\mathbf{Q}$ and a scaling by a factor 2 , then the centroids of the patterns satisfy the conditions for being an fcc/bcc grid in Definition 1.

Note that these patterns are not unique; there are 24 possibilities satisfying C1-C4 in each case. These patterns are obtained by rotation of the generator around the $x$-, $y$-, and $z$-axes by multiples of $\pi / 2$. This is in analogy with the patterns in the usual cubic grid. Often, resolution pyramids on the cubic grid is based on the generator $\{(0,0,0),(1,0,0),(0,1,0),(0,0,1),(1,1,0),(1,0,1),(0,1,1)$, $(1,1,1)\}$. However, any $2 \times 2 \times 2$ "cube" can be used as generator resulting in 8 possibilities.

From Table 1, the conclusion that none of the generators in Fig. 2 satisfy C2 can be drawn. The reason is that there is no pattern with ratio $(1: 4)$ or $(1: 2)$ for which the centroids form an fcc or bcc grid. 


\section{Multiscale Representation of Binary Images}

In this section, we will use the patterns obtained in the previous section to construct resolution pyramids. Each grid point is assigned either the value 1 or the value 0 . In the figures, the value 1 will correspond to object and the value 0 will correspond to background. We need, given a set of $1: \mathrm{s}$ in $\mathbb{G}_{T^{i}}$, some rules to decide which grid points should be assigned the value 1 in $\mathbb{G}_{T^{i+1}}$. Using the binary image in Fig. 4 as a running example, different rules will be examined. The images contains 18,995 (fcc) and 18,915 (bcc) object grid points. Observe that, in order to show the details in the different levels in the pyramid, the images are scaled. The shifting grid method and the intermediate grey-level method are generalizations of the methods in $[11,12]$.

\subsection{Simple Rules}

The logical rules OR and AND form the simplest pyramids.

OR If any grid point in a pattern in $\mathbb{G}_{T^{i}}$ has value 1 , then the corresponding grid point in $\mathbb{G}_{T^{i+1}}$ is set to 1 .

AND If all grid points in a pattern in $\mathbb{G}_{T^{i}}$ has value 1 , then the corresponding grid point in $\mathbb{G}_{T^{i+1}}$ is set to 1 .

The resolution pyramids obtained by using these rules on the objects in Fig. 4 are shown in Fig. 5. Note that for thin structures, the OR-rule is the only sensible choice, as otherwise the lower resolution levels will be empty.

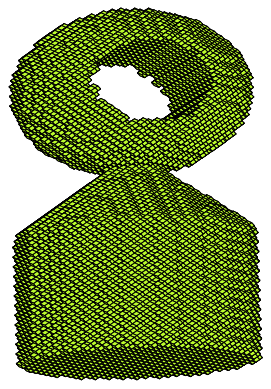

(a)

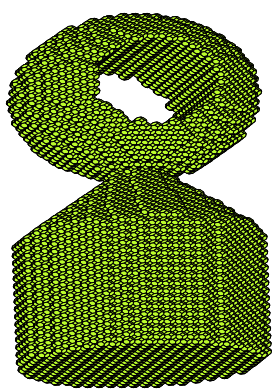

(b)

Fig. 4. A binary object on fcc (a) and bcc (b) used in the report

\subsection{The Shifting Grid Method}

In this method, the fact that there is an ambiguity when constructing the patterns is used. As mentioned in Section 3.2, there are 24 possible patterns all 


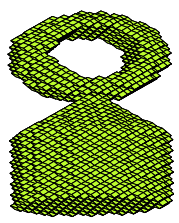

(a)

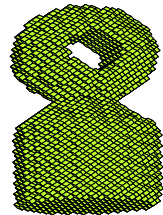

(g)

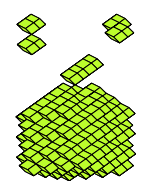

(b)

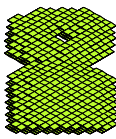

(h)

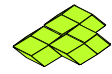

(c)

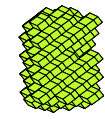

(i)

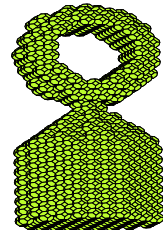

(d)

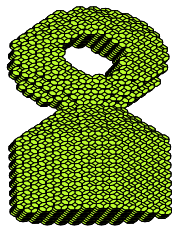

(j)
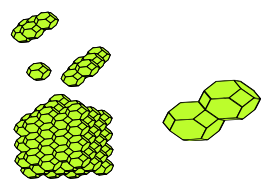

(e)

(f)
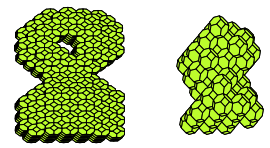

(k)

Fig. 5. Resolution pyramids consisting of $\mathbb{G}_{T}-\mathbb{G}_{T^{3}} ;$ (a)-(c) fcc, AND-rule; (d)-(f) bcc, AND-rule; (g)-(i) fcc, OR-rule; (j)-(l) bcc, OR-rule

giving the ratio $(1: 8)$. The information obtained by considering the remaining 23 possible patterns can be used to construct a stable multiscale representation. The idea is to use all the possible patterns in 24 different grids, denoted $\mathbb{G}_{T_{j}^{i}}$, where the subscript $j \in\{1, \ldots, 24\}$ denotes which one of the 24 different patterns that is used. When constructing the $\mathbb{G}_{T_{j}^{i+1}}$ images from $\mathbb{G}_{T_{j}^{i}}$, we use the OR-rule to produce all the 24 possible images that can be constructed. To combine these to one single image $\mathbb{G}_{T^{i+1}}$, the AND-rule is used: if all grid points at a corresponding position in $\mathbb{G}_{T_{j}^{i+1}}, j=1, \ldots, 24$ are 1 :s, then the grid point in $\mathbb{G}_{T^{i+1}}$ is set to 1 . This method is called "AND-ing the ORs" shifting grid method. Alternatively, by first using the AND-rule and then the OR-rule, a similar method called "OR-ing the ANDs" shifting grid method is obtained.

When implementing this method, it is not necessary to compute all 24 intermediate images. It is enough to scan the image once and for each grid point apply all the 24 patterns on the image in the previous level. By combining the OR and the AND-rule (or the AND- and the OR-rule), a more efficient shape preservation is obtained compared to the simple methods in Section 4.1. We get connected objects with a tunnel even in the lowest resolution.

Resolution pyramids of the objects in Fig. 4 using the AND-ing the ORs shifting grid method are shown in Fig. 6.

\subsection{The Intermediate Grey-Level Method}

As mentioned in the previous section, a scan through the image at level $i$ is enough to construct the image in level $i+1$ using the shifting grid method. The idea resulting in the intermediate grey-level method is to simulate the construc- 


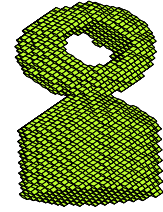

(a)

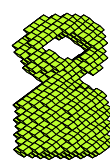

(b)

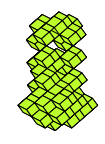

(c)

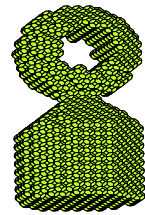

(d)
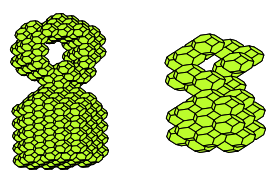

(e)

Fig. 6. Resolution pyramids consisting of $\mathbb{G}_{T}-\mathbb{G}_{T^{3}} ;(\mathrm{a})-(\mathrm{c})$ fcc, AND-ing the ORs shifting grid method; (d)-(f) bcc, AND-ing the ORs shifting grid method

tion of all possible patterns by using weights in a neighbourhood of each grid point. Suppose that $\mathbf{0}$ is included in all of the 24 generators. On the fcc grid, each first order neighbour is then included in 12 generators and each second order neighbour is included in 4 generators. On the bcc grid, each neighbour is included in 12 generators. These numbers will be the weights we use in the method. A mask is constructed consisting of the grid point and all neighbours of first and second order using these weights.

An intermediate grey-valued level $\mathbb{G}_{T}^{\prime}$ is constructed by placing the weighted mask at each a-point in the patterns in $\mathbb{G}$ and adding the weights of the mask positioned on a grid point with value 1 in $\mathbb{G}$. Now, we need to binarize $\mathbb{G}_{T}^{\prime}$ to achieve $\mathbb{G}_{T}$. The grey-level of a grid point in $\mathbb{G}_{T}^{\prime}$ is denoted $\Sigma$. A low value of $\Sigma$ indicates a small number of neighbours with value 1 . Thus, if $\Sigma$ has a low value, it would probably be set to 0 in the next level for most of the 24 patterns in an image using only one of the 24 patterns. If $\Sigma$ has a large value, it would probably be set to 1 . An intermediate value of $\Sigma$ indicates that it would be set to 0 for some of the patterns and to 1 for some patterns. To decide whether $\Sigma$ should be set to 1 or 0 , we state an extra criterion based on the values of the neighbours of $\Sigma$ in $\mathbb{G}_{T}^{\prime}$. If $\Sigma$ is larger than the average value in the neighbourhood consisting of first- and second-order neighbours, then it is set to 1 , otherwise to 0 .

\section{Criterion}

If $0 \leq \Sigma<64$ set $p$ to 0

If $64 \leq \Sigma \leq 128$ set $p$ to 1 if $\Sigma$ is larger than the average value in the first-order neighbourhood centered at $p$.

If $128<\Sigma \leq 192$ set $p$ to 1

The thresholds that are used corresponds to the thresholds in [11]; the sum of the mask values on the cubic grid is 64 and the thresholds used are 21 and 43 . The thresholds on the fcc and bcc grids are calculated by scaling these thresholds by using the sum of the mask values on the fcc and bcc grids, 192. The thresholds obtained by this procedure is 63 and 129. The method is, however, not very sensitive to changes of the threshold values. To get symmetric intervalls, we use the thresholds 64 and 128 instead. The values 60 and 124 or 68 and 132 would give similar results. Resolution pyramids using this method are shown in Fig. 7. 


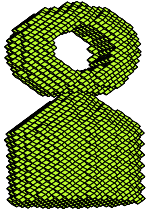

(a)

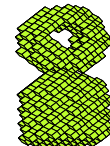

(b)

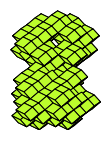

(c)

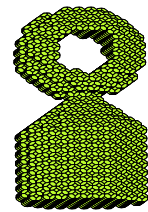

(d)
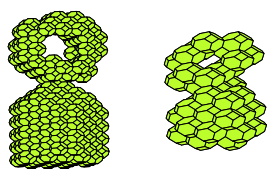

(e)

Fig. 7. Resolution pyramids consisting of $\mathbb{G}_{T}-\mathbb{G}_{T^{3}} ;$ (a)-(c) fcc, the intermediate greylevel image method; (d)-(f) bcc, the intermediate grey-level image method

Also in this case we get connected objects with a tunnel even in the lowest resolution level.

\section{Conclusions}

The most commonly used resolution pyramid on the hexagonal grid has ratio $(1: 7)$. One problem with this partitioning is that the grid is rotated by $\arctan \sqrt{3 / 5}$ for each level in the resolution pyramid. If the image is represented by a hexagonal grid in each level, the image will be transformed by a rotation which makes the resolution pyramid not suited for, e.g., comparing images captured at different resolutions. In contrary, no rotation is needed for the partitionings of the fcc and bcc grids suggested in this article. The resolution pyramid on the cubic grid has the same ratio as the resolution pyramids in this article, which makes methods for multiscale representations on the cubic grid easy to adapt to the fcc and bcc grids.

As expected, the AND- and OR-rules for multiscale representation give poor results. When using the AND-rule, to many grid points are set to 0 and when using the OR-rule, too many grid points are set to 1 . This implies that quality of the image (the topology preservation and the perceived shape) rapidly decreases when resolution is decreased. The perceived shape is better maintained using the shifting grid and the intermediate grey-level methods. A difference between the intermediate grey-level method and the shifting grid method is that when using the shifting grid method, each image obtained using the OR-rule is handled separately. This gives a slightly different result compared to when all possible patterns are handled simultanously using a mask. Also, when using the mask in the intermediate grey-level method, all possible positions of the patterns are considered, some of which might not be included in the shifting grid method. No one of these methods guarantees topology preservation of objects as changes of topology are unavoidable when the resolution is decreased. If we choose to maintain the connectedness of an object with a tunnel and decrease the resolution, then for each level of the resolution pyramid, the tunnel will be smaller than in the previous level. At some point, the tunnel will vanish. When the tunnels are 
big enough, the shifting grid and the intermediate grey-level methods have nice topology-preserving properties. They are also designed to remove the sensitivity to where the object is positioned in the image. It is also worth noticing that the intermediate grey-level method is faster, since a mask is used instead of applying 24 patterns at each grid point.

\section{References}

1. Matej, S., Lewitt, R.M.: Efficient 3D grids for image reconstruction using spherically-symmetric volume elements. IEEE Transactions on Nuclear Science 42 (1995) 1361-1370

2. Bell, S.B.M., Holroyd, F.C., Mason, D.C.: A digital geometry for hexagonal pixels. Image and Vision Computing 7 (1989) 194-204

3. Herman, G.T.: Geometry of Digital Spaces. Birkhäuser, Boston (1998)

4. Borgefors, G.: Hierarchical chamfer matching: A parametric edge matching algorithm. IEEE Transactions on Pattern Analysis and Machine Intelligence 10 (1988) 849-865

5. Rezaee, M.R., van der Zwet, P.M.J., Lelieveldt, B.P.F., van der Geest, R.J., Reiber, J.H.C.: A multiresolution image segmentation technique based on pyramidal segmentation and fuzzy clustering. IEEE Transactions on Image Processing 9 (2000) $1238-1248$

6. Rosenfeld, A.: Multiresolution image representation. In Levialdi, S., ed.: Digital Image Analysis. (1984) 18-28

7. Burt, P.J.: Tree and pyramid structures for coding hexagonally sampled binary images. Computer Graphics and Image Processing 14 (1980) 271-280

8. Lucas, D., Gibson, L.: Image pyramids and partitions. In: Proceedings $7^{\text {th }}$ international Conference on Pattern Recognition, Montreal. (1984) 230-233

9. Tanimoto, S.L., Crettez, J.P., Simon, J.C.: Alternative hierarchies for cellular logic. In: Proceedings $7^{\text {th }}$ international Conference on Pattern Recognition, Montreal. (1984) 236-239

10. Ahuja, N.: On approaches to polygonal decomposition for hierarchical image representation. Computer Vision, Graphics, and Image Processing 24 (1983) 200-214

11. Borgefors, G., Ramella, G., di Baja, G.S., Svensson, S.: On the multiscale representation of 2D and 3D shapes. Graphical Models and Image Processing 61 (1999) 44-62

12. Borgefors, G., di Baja, G.S., Svensson, S.: Multiresolution representation of shapes in binary images II: Volume images. In: Proceedings of Discrete Geometry for Computer Imagery (DGCI 1997), Montpellier, France. (1997) 75-86 